\section{Histoire Épistémologie Langage}

$42-2 \mid 2020$

Genèse, origine, récapitulation. Trân Đức Thảo face aux sciences du langage

\title{
De quelques intersections entre la philosophie linguistique de Trần Đức Thảo et la phénoménologie sémiotique
}

\section{Antonino Bondi}

\section{OpenEdition}

\section{Journals}

Édition électronique

URL : https://journals.openedition.org/hel/364

DOI : 10.4000/hel.364

ISSN : 1638-1580

\section{Éditeur}

Société d'histoire et d'épistémologie des sciences du langage

Édition imprimée

Date de publication : 31 décembre 2020

Pagination : 63-75

ISBN : 979-10-91587-13-6

ISSN : 0750-8069

\section{Référence électronique}

Antonino Bondì, « De quelques intersections entre la philosophie linguistique de Trần Đức Thảo et la phénoménologie sémiotique », Histoire Épistémologie Langage [En ligne], 42-2 | 2020, mis en ligne le 28 octobre 2021, consulté le 29 octobre 2021. URL : http://journals.openedition.org/hel/364 ; DOI :

https://doi.org/10.4000/hel.364

Ce document a été généré automatiquement le 29 octobre 2021.

\section{cc) (†)}

$\mathrm{HEL}$ is licensed under a Creative Commons Attribution-NonCommercial-NoDerivatives 4.0

International License 


\title{
De quelques intersections entre la philosophie linguistique de Trần Đức Thảo et la phénoménologie sémiotique
}

\author{
Antonino Bondì
}

\section{Introduction : I'héritage sémiotique et philosophique de Trân Đức Thảo}

1 Il semble aujourd'hui incontestable que l'intellectuel vietnamien Trần Đức Thảo appartient à une génération de philosophes $\mathrm{du} \mathrm{xx}^{\mathrm{e}}$ siècle qui a inlassablement questionné le langage en tant que champ problématique et véritable prisme épistémologique. Plus précisément, Thảo fait partie d'une cohorte de philosophes européens qui a interrogé la dimension sémiotique de l'expérience humaine en voulant échapper aux démarches dominantes de son époque. En effet, tant la pensée continentale que la philosophie analytique demeuraient, aux yeux du philosophe, plutôt problématiques. D'abord, la philosophie continentale: Trần Đức Thảo $\mathrm{y}$ appartient de façon claire, en étant un penseur marxiste d'orientation matérialiste et dialectique, mais aussi en tant que phénoménologue d'obédience husserlienne. Cependant, et malgré une telle appartenance, il essaiera pendant toute sa carrière de se démarquer d'une posture intellectuelle répandue parmi les continentaux, qui était celle de Heidegger à l'égard des savoirs positifs.

2 On sait que Heidegger a toujours conféré une place considérable au langage, en tant qu'objet d'étude et de réflexion; et cela au moins depuis le $\$ 32$ de Sein und Zeit. Le langage serait un véritable milieu garantissant l'authenticité de la forme de vie du Dasein : une condition originaire (ou précondition) de l'existence humaine. Pourtant, à un tel emplacement privilégié ne correspond pas une curiosité de la même ampleur pour le langage en tant que phénomène à objectiver du point de vue scientifique. 
Autrement dit, pour Heidegger, aucun savoir empirique, aucune connaissance spécialisée produite au sein des différentes branches des sciences du langage n'apparaissent décisifs pour comprendre l'essence du langage. La position de Trần Đức Thảo ne pourrait être plus radicalement contraire. D'abord parce qu'en tant que marxiste et matérialiste, il inscrit ses propres recherches dans une relation explicitement dialectique avec les savoirs positifs, afin de détecter les conditions matérielles qui permettent l'émergence d'une conscience linguistique et sociale. Ensuite, et encore une fois aux antipodes de la croyance de Heidegger, Thảo partage plutôt l'attitude d'autres philosophes d'orientation phénoménologique, et notamment Merleau-Ponty, Pos ou encore Maldiney. Afin d'approcher la complexité de l'expérience vécue, ces penseurs ont entamé dès le début de leurs travaux un échange de plus en plus étoffé et constant avec les disciplines empiriques, qu'il s'agisse des sciences humaines ou des sciences de la nature. Si pour les premières on pourra mentionner le dialogue intime entre Maldiney et le savoir psychiatrique (Ducard 2015), en ce qui concerne les sciences de la nature, la référence incontournable est le cours au Collège de France de Merleau-Ponty, consacré au concept de nature (Merleau-Ponty 1995). Le philosophe français y nouera un véritable dialogue épistémologique pointu avec des disciplines hétérogènes, telles la biologie et l'éthologie, la théorie de l'information et la cybernétique, mais aussi la psycholinguistique et la linguistique de Saussure et Jakobson.

3 Mais Thảo se démarque aussi du cadre théorique du linguistic turn qui a orienté la philosophie analytique du langage, allemande et surtout anglo-américaine, car il ne s'intéresse pas à l'examen des engagements ontologiques des expressions linguistiques, à une critique épistémique du langage ou à une thérapeutique logique clarifiant les deux questions précédentes. Pour le philosophe franco-vietnamien, le langage n'est pas un objet autonome tel qu'il est conçu par la koinè analytique du début du $\mathrm{xx}^{\mathrm{e}}$ siècle ; d'ailleurs, il ne représente pas non plus une clé purement spéculative pour comprendre la place de l'homme dans l'ordre du cosmos. En adhérant à une orientation fortement matérialiste, Thảo conçoit au contraire le langage comme un phénomène à la fois matériel et symbolique, c'est-à-dire à la fois biologique et intrinsèquement sociohistorique. Cette double essence, pour reprendre une expression de saveur saussurienne, fait du langage l'outil anthropologique par lequel on pourrait saisir et décrire la dimension symbolique de la forme de vie proprement humaine.

Ces considérations générales s'avèrent nécessaires, car elles poussent à expliciter l'orientation de cet article. Ici on ne prétendra pas reconstruire les propos, ou encore moins l'architecture globale de la théorie de Thảo. On visera plus modestement à s'interroger sur son anthropologie sémiotique, pour discuter de certaines intersections que les propositions de Thảo peuvent entretenir avec des perspectives contemporaines en sémiotique. Par conséquent, nous focaliserons notre attention sur ce que nous avons défini ailleurs comme une phénoménologie sémiotique (Coquet 2007 ; Bondì 2015 et 2018 ; Bondì et al. 2016). En fait, au cours des dernières décennies, de nombreuses recherches en sémiotique et en philosophie du langage ont fait preuve d'un intérêt renouvelé pour les perspectives phénoménologiques, gestaltistes et matérialistes issues des philosophies (et des linguistiques) du $\mathrm{xx}^{\mathrm{e}}$ siècle. En particulier, la phénoménologie sémiotique vise à comprendre et à décrire le statut de ce qui fait signe dans ses phases diverses (c'est-à-dire hétérogènes mais complémentaires) d'apparition, de stabilisation, d'emploi et de déformation. Il s'agit d'une perspective qui s'intéresse au détail de la 
singularité d'un objet de sens, langagier ou plus largement sémiotique. Cependant, la focalisation sur la singularité de l'objet langagier présuppose l'hypothèse selon laquelle toute détermination des signes est d'emblée captée ou saisie de façon dynamique dans la trame et dans la stratification des actions mises en œuvre par les sujets et par les communautés. Sujets et sociétés constituent, en reprenant un autre adage saussurien, les acteurs principaux des métamorphoses constantes des formes ainsi que des plans et des champs de sémiotisation et d'expression dans lesquelles les formes elles-mêmes se constituent (Cadiot \& Visetti 2001 ; Visetti \& Cadiot 2006 ; Rosenthal \& Visetti 2010).

Voulant croiser une étude de ce qui fait signe dans ses phases d'apparition, de stabilisation-institutionnalisation, d'emploi et de déformation incessante par les sujets et les communautés, la phénoménologie sémiotique tâche de tenir ensemble, dans un canevas descriptif hétérogène, les principes et les dynamiques de constitution des expériences de sens. C'est dans ce contexte que nous avons ailleurs essayé de faire résonner une linguistique/sémiotique textuelle (à orientation pragmatique) avec une proposition phénoménologique, afin d'articuler les différentes phases (matérielles et symboliques) de la conscience sémiotique (Bondì et al. 2016 ; Piotrowski \& Visetti 2015 ; Bondì \& La Mantia 2015). Si, en reprenant la leçon de Merleau-Ponty, la sémiose se constitue comme un champ dynamique de forces et de formes, et si elle se produit comme reprise perpétuelle et comme transformation incessante des formes et des consciences sémiotiques, maintes questions se posent: quelles sont les dynamiques matérielles et psychiques d'un tel mouvement génétique des consciences sémiotiques? Peut-on sérieusement penser en termes de genèse des formes, sans tenir compte de la socialité du sens et de la nature fortement sémiotique de la perception, comme nous indique l'approche phénoménologique de Merleau-Ponty? Et, si l'on accorde ce pouvoir sémiogénétique à la perception, comment concevoir le déploiement social et institutionnel, sans oublier le nécessaire rôle du vécu de l'expérience, qui traverse toute construction sémiotique? En effet, dans le cadre phénoménologique, et pour Merleau-Ponty en particulier, la perception fonde la sémiose en tant que celle-ci est originairement ancrée dans les faits perceptifs, et, en même temps, la perception est déjà expressive et significative dès le début (Merleau-Ponty 1945 et 1969). Dans le cadre de cette contribution, on se limitera à esquisser quelques aperçus de cette phénoménologie sémiotique d'inspiration merleau-pontienne et, par ce biais, à observer comment les recherches sur l'origine du langage et de la conscience chez Trần Đức Thảo peuvent en représenter un élément pour ainsi dire rétrospectivement archéologique.

6 En effet, parmi les propositions de Thảo en matière de théorie du langage, tant sa conception de la constitution indicative et ostensive de l'expérience sémiotique (à savoir l'idée d'une origine gestuelle de la sémiose), que certaines pages sur la formation de la conscience sémiotique, nous permettent de tenir ensemble, dans une structure chiasmatique, la dimension perceptive de la sémiose et la nature sociale et sémiotique de la perception. On explorera ce terrain problématique.

\section{1 Éléments de phénoménologie sémiotique : la parole comme flux héraclitéen}

7 La phénoménologie sémiotique se présente comme une critique des approches objectivistes en linguistique et aussi comme une mise en discussion des formes 
d'idéalisme qui ont affecté d'innombrables théories sémiolinguistiques. Elle reprend une indication merleau-pontienne et s'interroge sur la cartographie des relations entre le phénomène de la prise de parole, la ou les subjectivités - ou la constitution d'identités subjectives - et la socialité du sens.

D'abord, la prise de parole: de quoi parle-t-on? On entend par parole tout phénomène langagier qui se présente à la conscience des sujets parlants comme phénomène expressif, étalé et toujours déployé sur des plans de manifestation sensible, que l'on peut appeler, en adoptant le lexique de la sémiotique structurale hjelmslévienne, des plans d'expression. Le caractère principal de tout phénomène expressif est de nous faire signe. Ainsi, il nous incite à construire immédiatement une réponse. Autrement dit, un phénomène expressif, d'un point de vue phénoménologique, se définit comme un signe s'adressant toujours à quelqu'un, à nous, qui nous engage, convoque, implique et motive des actions qui sont conséquentes - ne serait-ce que sous la forme du silence, du refus, de l'incompréhension. Deux choses sont à retenir dans cette première définition du phénomène de la parole :

i. une non-distinction descriptive, épistémologique et ontologique que la notion et le phénomène de parole comporte, du moins par rapport au format qu'elle incarne et expérimente ;

ii. un paradoxe constitutif à propos du qui fait expérience de la parole et du comment (à savoir les modalités, les modes d'existence, la circulation et la transmission, ainsi que ses limites).

Partons du premier point. Soutenir que la parole consiste en tout phénomène langagier signifie ici contester l'idée (d'ailleurs légitime) qu'il y aurait quelque chose comme des formants ou des formats grammaticaux et/ou syntaxiques, ainsi que des formants sémantiques qui seraient donnés à priori. Autrement dit, on conteste la possibilité de concevoir les formants comme préalables à l'événement expressif lui-même, en dehors de l'immanence de la prise de parole. Traditionnellement, les formants dépassent leur simple vocation d'objet épistémologique et d'outil de classement pour être pensés comme des éléments donnés a priori, éventuellement stockés dans nos mémoires ou au sein des dispositifs culturels de récolte de savoir (tels les dictionnaires, les encyclopédies etc.). Si l'on suit cette tradition, les formants et les formats pourraient prétendre à être élevés au rang de seuls objets scientifiques objectivables, et par là à être à même de rendre compte du sémantisme et de l'épaisseur sémiotique d'une forme dotée de sens. La position phénoménologique ne conteste pas - il faut être clair - la légitimité épistémologique des notions de formants ou des formats, très utiles dans un cadre sémiotique. En se rapprochant d'une démarche déjà explorée par les théories énonciatives, on prend plutôt le parti de la parole, ce qui implique, plus modestement, de garder une attitude pluraliste à l'égard des différents formants linguistiques et langagiers (des morphèmes ou submorphèmes jusqu'aux formations discursives et polysémiotiques plus vastes). Mais prendre le parti de la parole signifie aussi poursuivre un intérêt fondamental (et non parasitaire) pour le flot de la parole, qui est un véritable refoulé dans un grand nombre de théories du langage.

En suivant les indications de Saussure depuis ses cours de linguistique générale à Genève (Saussure 2002), on entend par flot de la parole une activité incessante et ininterrompue de parler, anonyme et hyper-singulière. Il s'agit, comme Saussure le répète à plusieurs reprises, de la source de variation perpétuelle propre à l'instinct linguistique : c'est une masse de productions langagières à la fois très singulière et intimement sociale et collective. Le flot de parole est singulier car il est profondément 
lié à $m a$ propre expérience du monde et du sens : une expérience qui est dès le début pour ainsi dire syncrétique et qui m'affecte toute ma vie. Mais le flot de la parole est en même temps intimement social et collectif, car il relève de la masse parlante et anonyme, sans laquelle l'activité de parler et la parole seraient tout simplement inconcevables et inexistantes. Ce n'est pas un hasard si Saussure a affirmé de façon très explicite que le langage est à saisir comme deux choses à la fois : une faculté, à savoir un instinct propre à l'espèce humaine, qui est un instinct praxéologique; et une activité d'éducation individuelle, un entraînement à la socialité et à la réglementation conventionnelle. Ainsi, il écrit un passage qui synthétise de façon très éloquente ce que nous venons de résumer : «dans l'acte de langage, la langue tire à la fois son application et sa source unique et continuelle [...] le langage est à la fois l'application et le générateur continuel de la langue, [...] la reproduction et la production » (Saussure $2002: 129$ ).

Donc, si l'on s'en tient à ses premières remarques, une perspective phénoménologique en sémiotique critique l'idée d'une forme immanente et préalable aux événements expressifs, et propose une méthode d'observation et d'analyse des émergences de formes, dès qu'elles sont captées, perçues et pendant les phases où elles se stabilisent à travers une pluralité de normes, de modalités et même de simples coïncidences.

Le cadre saussurien a été repris par Merleau-Ponty (et Pos) pour travailler de façon plus approfondie et cohérente le rôle du sujet parlant dans un dispositif sémio-linguistique de type expérientialiste (Bondì 2014 ; Coquet 2007 ; Piotrowski \& Visetti 2015). C'est par ce biais que l'on rencontre les méditations de Thảo, dès que l'on veut travailler sur la nature paradoxale du chiasme entre socialité du sens et subjectivité de l'expérience. En fait, comme nous avons écrit plus haut, un phénomène expressif est caractérisé par son déploiement dans des plans de manifestation sensible. La formation des événements expressifs requiert une espèce de convocation permanente à nous engager. Si l'on s'en tient à une telle définition, il s'avère nécessaire de croiser la réflexion sur la prise de parole avec l'étude des conditions matérielles et corporelles de son émergence ou, en termes phénoménologiques, de son apparition.

\section{L'anthropologie sémiotique de Thảo et l'archéologie gestuelle du sens}

13 La tradition scientifique de la linguistique occidentale a été incontestablement influencée par la métaphysique occidentale implicite dans tout savoir spécialisé. Cette influence a déterminé une conception des phénomènes linguistiques à partir de leurs dimensions et aspects productifs. Dans un certain sens, l'expression prise de parole reflète elle aussi partiellement cette habitude : à l'origine de la parole il y aurait un sujet substance ou activité mentale peu importe - qui communique ses intentions de façon consciente, et cela même lorsqu'il est travaillé par des instances inconscientes. Un tel sujet, pour ainsi dire, programme et réalise son intention de dire, de façon transparente, et transmet son message à un interlocuteur. Ce message aurait par conséquent un format plus ou moins standardisé et recevable par l'autre: un format plus ou moins clair, déterminé et compact, que l'on peut interpréter sur la base des connaissances et des intentions de l'interprète. Un examen, même rapide, de nombreux dispositifs en linguistique théorique confirmerait cette habitude conceptuelle qui a affecté implicitement la théorie linguistique. Songeons par exemple au cas extrême de rejet de la subjectivité en théorie du langage, c'est-à-dire à Chomsky, qui a toujours refusé de 
donner à l'intention de dire tout statut d'objet sémiotique ou linguistique. Malgré un tel refus, par le biais du maintien de la séparation nette entre compétence et performance, on assiste à la résistance d'un présupposé pour ainsi dire philosophique (et même métaphysique), à savoir l'idée d'une primauté de la production énonciative de la part des sujets - dans le cas de Chomsky (que nous évoquons ici de façon caricaturale), c'est un parlant natif idéalisé. De ce point de vue, certaines des réflexions contenues dans les Recherches sur l'origine du langage et de la conscience de Thảo représentent des antidotes à ce genre de présupposés métaphysiques; par conséquence, une approche à la fois phénoménologique et sémiogénétique ne peut pas les négliger ${ }^{1}$.

14 En évoquant une espèce de scène anthropogénétique primaire, le philosophe reconstruit en effet la dynamique de constitution du mouvement indiciel, qu'il appelle parfois de façon plus appropriée le véritable mouvement de l'indication. De façon plutôt radicale, Thảo $(1973: 16)$ pense à une typologie de mouvement au statut de forme fondamentale: c'est le mouvement en arc de cercle, qui représente à ses yeux le geste sémiotique par excellence. D'abord, pourquoi parle-t-on de geste sémiotique? Une première réponse réside dans le fait qu'il s'agit d'une relation originairement à trois termes, dont la finalité - comme, par exemple, dans le cas des signes chez Peirce - est la construction d'une série de schématisations et d'inférences à utiliser pour se repérer dans l'environnement. Ensuite, Thảo affine la réponse et présente une qualification spécifique de l'orientation sémiotique de ce geste fondamental, qui est dès le début orienté par des finalités pragmatiques et herméneutiques complexes. C'est le geste du pointage, que l'on serait tenté de définir en termes d'un complexe inférentiel. En effet, l'objet indiqué et éventuellement vocalisé par le pointage constitue un pôle d'attraction engendrant une prolifération de gestes d'attention plus ou moins partageables. Ainsi, la perception d'un objet externe est contrainte par un travail proprement social et intersubjectif: intersubjectif car ce qui se joue est la mobilisation des intérêts et des attentions ; social car le pointage est toujours encadré et déterminé par la différence et la spécificité des pratiques où a lieu la capture perceptive même de l'objet. Autrement dit, l'acte fondamental qualifiant ce type de perception sémiotique, c'est-à-dire le pointage, se conçoit comme un phénomène d'inter-subjectivisation du geste d'indication. Comme écrit le philosophe : «il s'agit d'un acte de guidage, dont la seule différence avec le geste de l'indication en arc de cercle est que le sujet humain se tient à distance " (Thảo $1973: 16$ ).

15 Mais Thảo ajoute un passage crucial, poussant dans une direction plus sémiolinguistique l'archéologie indicielle de la scène du sens. En effet, par le biais du pointage, le processus complexe de l'indication se construit sur la base de pressions attentionnelles diverses et conjointes, c'est-à-dire selon une pragmatique reconnaissant le rôle exercé par les autres. Autrement dit, la scène du sens s'inscrit dans une composition d'arènes interactionnelles primaires. Cependant, Thảo pense à la configuration de l'autre dans le processus d'indication non pas en simples termes d'attraction ou de répulsion attentionnelles, mais comme le résultat d'une activité d'appel. Si l'on suit un raisonnement étonnant et perspicace de Thảo, cet appel se comprend comme la convocation nécessaire d'une altérité, celle-ci à son tour ne pouvant entrer en relation avec l'objet que par le biais d'une scène aussi complexe. Comme suggère Thảo (ibid.: 21-22) lui-même :

En définissant le geste comme un guidage à distance, nous avons insisté jusqu'ici sur sa forme. En réalité le mouvement du guidage ne consiste pas simplement à tracer une direction, il comporte essentiellement la fonction d'appel. Le geste de 
l'indication, comme guidage à distance, est un appel au travail sur l'objet indiqué. Or, en tant qu'appel, il se complète tout naturellement par la forme normale de l'appel, à savoir la forme vocale. conscience sémiotique. C'est pour cette raison que le sujet peut se donner: il n'est concevable qu'à partir d'un très nourri et articulé théâtre de la conscience ${ }^{2}$. Encore Thảo (ibid.: 25) :

On voit que la forme du geste se constitue comme la synthèse de deux moments réciproques de la forme en arc de cercle : le moment où le sujet est donneur et celui où il est receveur de l'indication. Il est donc maintenant, dans un seul et même mouvement, à la fois donneur et receveur. Bref, en raison de la situation du retardataire, le mouvement de l'indication dans sa totalité, geste et voix, revient sur lui-même, de sorte que le sujet s'indique à lui-même à partir des autres avec lesquels il s'identifie.

Par conséquent, Thảo définit le signe en termes de résultat pragmatique et de prise de conscience. Cette prise de conscience, articulée en phases différentes, se réalise à travers un double processus du théâtre de la signification-un processus à la fois d'extériorisation comme d'intériorisation. De ce point de vue, on peut donc affirmer que pour Thảo la signification relève d'un processus complexe de thématisation, celleci coïncidant avec la conscience. Ainsi écrit Thảo (1973 : 30-31) :

Nous voyons ainsi la prise de conscience, dans son mouvement originaire, se réaliser en trois moments. Tout d'abord surgit, dans une situation de retard, une prise de conscience sporadique, comme éclair de conscience. Elle a pour fonction d'inciter le sujet à surmonter son propre retard pour se mettre au niveau du mouvement social. Puis vient la prise de conscience collective, qui permet une première généralisation du signe en le rendant disponible pour le groupe en dehors des situations de travail urgent. Enfin arrive la prise de conscience individuelle, qui achève la généralisation du signe en le rendant constamment disponible pour l'individu. Le sujet peut désormais utiliser systématiquement ce signe devenu conscient en lui-même, pour agir sur lui-même et les autres, mobiliser et diriger les énergies du groupe pour l'appropriation de l'objet. Tel est évidemment, dès l'origine, le fondement du rôle pratique de la conscience.

Dans cette évocation - très partielle - de certaines propositions de Thảo, trois éléments nous semblent importants à retenir :

i. la dimension d'appel propre de la formation de la scène où l'indication a lieu, qui présuppose une socialité intrinsèque de toute pratique de sens ;

ii. la prise de conscience comme mouvement d'une généricité collective et anonyme en montée thématique en situation ;

iii. cette montée thématique est possible parce que la typologie des objets créés, c'est-à-dire les signes, relève des dynamiques de disponibilité et d'appropriation qu'en font les sujets, ou, en utilisant les termes de la phénoménologie sémiotique et de la théorie de la Gestalt, en termes de transposabilité.

19 Si la parole en tant qu'événement expressif est à qualifier comme un processus dynamique de thématisation dont l'émergence relève d'une pluralité de régimes, il faut s'interroger sur la nature de ces régimes. De ce point de vue, l'interrogation sur le concept de forme sémantique, central dans l'approche que j'ai proposée plus haut, demeure importante. 


\section{Pour conclure : une piste entre phénoménologie sémiotique et herméneutique textuelle}

La notion de forme sémantique a fait son apparition dans les champs des sciences du langage à une époque assez récente, au sein de la linguistique textuelle. D'apparence intuitive, le syntagme forme sémantique recouvre en effet un double statut : d'une part, il semble jouer le rôle d'un simple outil descriptif visant la reconnaissance des formes à partir de leurs dimensions méréologiques et mélodiques. D'autre part, cette notion dévient le concept pivot d'une théorie du langage et de la sémiose à vocation phénoménologique et dynamiciste, étroitement liée et pourtant dans un rapport dialectique avec la pragmatique post-wittgensteinienne, l'herméneutique textuelle et la refonte du structuralisme biodynamique de René Thom (Cadiot \& Visetti 2001 ; Bondì 2018).

21 Le champ problématique de la forme sémantique est construit à partir du thème de l'identification et de la reconnaissance des unités. Comment reconnaît-on une forme ou une unité sémantique? Quels ordres ou niveaux de description de l'analyse linguistique implique-t-elle? Quels en sont les caractères définitoires et, par conséquent, les procès de constitution? L'étude des morphologies nécessite une inversion méthodologique et théorique. S'il est possible de décrire les unités par une multiplicité de rapports, procès et composants les constituant, il faut repenser la notion de forme ou de Gestalt en un sens dynamique. En d'autres termes: parler de morphologies équivaut à parler de Gestalten fluctuantes et en évolution pérenne, se structurant selon une multiplicité d'éléments qui s'intègrent réciproquement, mais pas nécessairement dans une relation de type hiérarchique. Une unité émerge par un ensemble d'interactions différenciées de plusieurs composants, dont les relations ne sont pas nécessairement hiérarchisées mais plutôt des relations de synchronicité ou de simultanéité. Ainsi, l'analyse d'une forme sémantique peut passer par des stratégies descriptives diverses :

i. une description paradigmatique à travers l'insertion dans un répertoire de formes ;

ii. une description syntagmatique focalisant plutôt sur la concaténation de formes ;

iii. une description herméneutique, faisant émerger le résultat du parcours de constitution ou reconstitution de la forme;

iv. une description référentielle, qui souligne, quant à elle, les tissus relationnels des formes linguistiques aux formes non langagières.

Dès lors, la question du sens linguistique semble ne pas pouvoir faire l'économie des rapports avec l'insertion au sein des dispositifs textuels d'une part et des pratiques interprétatives orientées selon les contextes de l'autre. Cette double insertion, finalement, est le caractère principal des modèles phénoménologiques de l'activité du langage, qui s'avèrent en mesure de rendre compte de l'expérience sémiogénétique. Deux nœuds problématiques seraient à approfondir :

i. l'entrelacs constitutif entre langage (ou langues) et expériences sémiogénétiques, en focalisant sur le chiasme entre perception, sémiose et socialité du sens ;

ii. l'élaboration et la description des parcours de constitution de formes proprement linguistiques.

23 Il s'agit d'un cadre théorique qui insiste sur la reprise de thèmes de la tradition gestaltiste et phénoménologique, et dont la finalité explicite est la description en style phénoménologique de la valeur linguistique, à saisir dans sa continuité avec l'expérience 
sensible et pratique, sans pour autant oublier les exigences descriptives de la linguistique textuelle et interprétative. Ainsi, il serait envisageable de décliner ensemble l'émergence de la singularité du phénomène linguistique, dans sa dynamique interne, avec sa dimension plus textuelle, discursive, énonciative. Le concept de forme sémantique devient le pôle d'attraction de ces deux tensions constitutives de la linguistique: d'une part elle est une sorte de phénoménologie herméneutique de la singularité linguistique (l'acte de parole de la tradition saussurienne); de l'autre, elle est destinée à devenir une récognition des attestations textuelles, avec ses sédimentations formelles, qui constituent l'expression de la productivité langagière humaine.

Du point de vue méthodologique, la procédure descriptive envisage non pas le repérage d'invariants linguistiques de tout genre, mais à une mesure de l'extension d'usage des mots, pour en saisir l'instabilité constitutive, les ouvertures plastiques et génériques qui font résonner le langage avec un horizon pratique et sémiotique en transformation constante. C'est sur ce fond de généricité instable que les formes linguistiques se déploient, afin de se stabiliser et en même temps de se laisser rouvrir à de nouvelles déterminations. Il s'agit de penser aux processus de signification comme à des déterminations ouvrant non pas seulement sur des horizons de sens, mais toujours à la recherche d'une reprise de nouvelles déterminations en attente.

D'un point de vue plus théorique, on conçoit l'activité de langage comme une perception et/ou construction de formes, sur la base d'une analogie constructive entre vie perceptive et vie linguistique. Aussi, derrière quelque chose qui se présente comme un ensemble de niveaux stables manifestés dans les langues se cache une dynamique de déploiement et d'interaction entre divers régimes en coalescence. D'un côté, il faut poser cette première question : de quelle façon peut-on comprendre le langage comme activité de construction dynamique de Gestalten sémiotiques, sans pour autant réduire le concept de Gestalt à la seule dimension topologique et spatiale? De l'autre côté, il faudra reprendre la problématique du corps en tant que matrice de construction sémantique, dans le sillage de la tradition gestaltiste et phénoménologique, ainsi que comme lieu de l'union et du travail simultané de perception, action et expression. La phénoménologie, sur laquelle on prend appui, pense au corps comme toujours déjà capté dans une trame d'interrelations denses de signification; la corporéité constitue une trame intentionnelle qui œuvre par reprises incessantes de ce qui a été déjà thématisé, par immersion dans les horizons et par les motifs qui y sont rattachés à l'état aléatoire, latent (Bondì à paraître). En ce sens, la logique des formes sémantiques est une logique de l'expression, c'est-à-dire une logique des temps et des dynamiques d'organisation, d'extériorisation et de reprise de l'expérience verbale elle-même. De plus : la langue elle-même devient une saisie du monde et en même temps une saisie du discours d'autrui dans le monde: une pratique de co-expression dans laquelle le rapport entre sens et référence s'articule comme une relation d'extension réciproque plutôt que de simple correspondance. Dans ce cadre, la notion d'expérience fait son retour au sein de la théorie linguistique, comme Cadiot et Lebas (2003:5) ont remarqué :

non seulement les options phénoménologiques sont d'une grande compatibilité avec l'observation de toutes les strates et les dimensions du langage, mais encore elles livrent une solution générale au problème de l'articulation sens-référence (...). L'essence de cette solution réside dans un retour à l'expérience : le monde est une constitution compatible avec l'expérience parce qu'il est constitué par l'expérience. 
La conséquence de ce point de vue théorique est une tentative de formuler à nouveaux frais ce qu'on entend par situation discursive concrète:

la solution générale de l'articulation sens-référence est alors énonçable avec une extraordinaire simplicité: les objets de la parole sont propres à l'activité linguistique en tant qu'ils sont en partie constitués par la dynamique langagière, mais sont aussi les mêmes que ceux auxquels le langage réfère. Ceci cesse précisément d'être paradoxal dès le moment que le réfèrent n'a d'autre essence que ses propriétés extrinsèques. Il $\mathrm{y}$ a ainsi, en contradiction avec les thèses "dualistes», continuité entre le monde conçu par la pratique langagière et le monde conçu par les autres pratiques. (ibid.)

Ainsi, il devient possible de repenser l'articulation sens-référence, en constatant que «la signification se fonde dans, et est fondée par, les termes mêmes de la conceptualisation, dans le même temps que le langage redevient une pensée particulière, la parole une expression, la langue une pratique » (ibid. : 6). On constate la présence d'un lien profond entre l'activité de signification en tant que pensée, l'acte de parole en tant qu'expression et la langue en tant que pratique. Précisons cet aspect et retrouvons les suggestions de Trần Đức Thảo, qui s'avèrent actuelles dans le panorama contemporain. En effet, si le rapprochement entre langue et pratique d'expression vise à construire une perspective linguistique orientée dans un sens phénoménologique, il est nécessaire de mettre en relief les relations entre corporéité et dimensions langagières. Ce rapprochement permettrait en effet de cerner en même temps deux choses : d'une part, la spécificité linguistique par laquelle formuler une hypothèse forte sur les relations entre faculté de langage et faculté perceptive; d'autre part, une spécificité linguistique qui traverse et imprègne toute strate du langage, et sur quoi construire l'idée de forme sémantique. Au cœur de l'idée de conscience langagière que la phénoménologie sémiotique poursuit aujourd'hui, on trouve l'idée d'une prise de conscience située et mouvante, par le jeu social d'une gestualité primordiale produisant un théatre complexe du sens, de l'activité des corps et des esprits, comme nous l'apprend encore aujourd'hui Trần Đức Thảo.

\section{BIBLIOGRAPHIE}

Bondì, Antonino. 2014. L'expérience de la parole : le thème du sujet parlant. Revue-Texto 19.1 [http://www.revue-texto.net/index.php?id=3443, consulté le 18/02/2021].

Bondì, Antonino. 2015. Pour une anthropologie sémiotique et phénoménologique. Le sujet de la parole entre cognition sociale et valeurs sémiolinguistiques. Intellectica $63: 125-148$.

Bondì, Antonino. 2018. L'expression entre expérience et physionomies du sens. Éléments pour une phénoménologie sémiotique. Phenomenology and Structuralism. Acta Structuralica - International Journal for Structuralist Research $\mathrm{n}^{\circ}$ spécial 1 dir. par Rossana De Angelis \& Simone Aurora, 71-88.

Bondì, Antonino. À paraître. L'idea di motivo, dalla filosofia linguistica di Merleau-Ponty alla semantica contemporanea. Atti del primo convegno internazionale CISPELS. 
Bondì, Antonino. \& Francesco La Mantia. 2015. Phenomenology and Semiotics. Crossing-over Perspectives. Metodo. International Journal of Phenomenology and Philosophy 3.1 : 7-20.

Bondì, Antonino., David Piotrowski \& Yves-Marie Visetti. 2016. Linguistique et phénoménologie : un entrelacs. Metodo. International Journal of Phenomenology and Philosophy 4.2 : 267-308.

Cadiot, Pierre \& Franck Lebas. 2003. La constitution extrinsèque du référent : présentation. Langages $150: 3-8$.

Cadiot, Pierre \& Yves-Marie Visetti. 2001. Pour une théorie des formes sémantiques. Motifs, profils, thèmes. Paris : Puf.

Coquet, Jean-Claude. 2007. Phusis et logos. Une phénoménologie du langage. Paris : Presses universitaires de Vincennes.

Ducard, Dominique. 2015. De deux discours l'un. Phénoménologie de l'existence et sémiotique de la présence. Metodo. International Journal of Phenomenology and Philosophy 3.1 : 147-164.

Merleau-Ponty, Maurice. 1945. Phénoménologie de la perception. Paris : Gallimard.

Merleau-Ponty, Maurice. 1969. La prose du monde. Paris : Gallimard.

Merleau-Ponty, Maurice. 1995. La nature. Notes. Cours du Collège de France suivi des résumés de cours correspondants. Paris : Seuil.

Piotrowski, David \& Yves-Marie Visetti. 2015. Expression diacritique et sémiogenèse. Metodo. International Journal of Phenomenology and Philosophy 3.1 : 63-112.

Rosenthal, Victor \& Yves-Marie Visetti. 2010. Expression et sémiose pour une phénoménologie sémiotique. Rue Descartes 70 : 24-60.

Saussure, Ferdinand de. 2002. Écrits de linguistique générale établis et édités par Simon Bouquet et Rudolf Engler ; avec la collaboration d'Antoinette Weil. Paris : Gallimard.

Sériot, Patrick. 1999. Structure et totalité. Les origines intellectuelles du structuralisme en Europe centrale et orientale. Paris : Puf.

Thảo, Trần Đức. 1973. Recherches sur l'origine du langage et de la conscience. Paris : Éditions sociales. Thảo, Trần Đức. 1974. De la phénoménologie à la dialectique matérialiste de la conscience (I). La Nouvelle Critique 79-80 : 37-42.

Visetti, Yves-Marie \& Pierre Cadiot. 2006. Motifs et proverbes. Essai de sémantique proverbiale. Paris : Puf.

\section{NOTES}

1. Cf. Thảo 1973 et 1974.

2. L'expression «théâtre de la conscience » n'est pas utilisée de façon explicite par Thảo, mais il s'agit d'une idée répandue à l'époque dans de nombreux milieux philosophiques, psychologiques et linguistiques. Il s'agit, par exemple, d'une idée partagée par Bakhtine et Vološinov, et même par Vygotski et la linguistique soviétique de l'époque. Cf. Sériot 1999. 


\section{RÉSUMÉS}

L'article vise à explorer les entrecroisements possibles entre la phénoménologie sémiotique contemporaine et la scène originaire du sens, telle qu'elle a été conçue par Trần Đức Thảo. Dans les deux approches est développée une même conception de la conscience sémiotique comme opération de pointage complexe et de construction de scène énonciative. Phénoménologie sémiotique et matérialisme dialectique retrouvent dans la conception de la sémiose comme processus dynamique social une source de convergence thématique insoupçonnée.

The article aims to explore the possible intersections between contemporary semiotic phenomenology and the original scene of meaning, as conceived by Trần Đức Thảo. In both approaches, the same conception of semiotic consciousness is developed as an operation of complex pointing and enunciative scene construction. Semiotic phenomenology and dialectical materialism find, in the conception of semiosis as a dynamic social process, a source of unsuspected thematic convergence.

\section{INDEX}

Mots-clés : phénoménologie, sémiotique, matérialisme, conscience, praxis

Keywords : phenomenology, semiotics, materialism, consciousness, praxis

\section{AUTEUR}

\section{ANTONINO BONDİ}

Università degli studi di Catania, Catane, Italie 